Weber - Markenpolitik des Bankbetriebs 
OIKOS - Studien zur Ökonomie - Band 32

Herausgegeben von Dr. Stephan M. Hess 
Markus Weber

\section{Markenpolitik des Bankbetriebs}

Grundlagen und empirische Studien

\section{GABLER}


Die Deutsche Bibliothek - CIP-Einheitsaufnahme

\author{
Weber, Markus: \\ Markenpolitik des Bankbetriebs: Grundlagen \\ und empirische Studien / Markus Weber. - \\ Wiesbaden: Gabler, 1992 \\ (Oikos; 32) \\ ISBN 978-3-409-14802-3 \\ DOI 10.1007/978-3-322-87991-2 \\ NE: GT

Der Gabler Verlag ist ein Unternehmen der Verlagsgruppe Bertelsmann International.

(C) Betriebswirtschaftlicher Verlag Dr. Th. Gabler GmbH, Wiesbaden 1992 Lektorat: Karlheinz Müssig

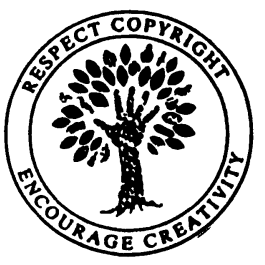

Das Werk einschließlich aller seiner Teile ist urheberrechtlich geschützt. Jede Verwertung außerhalb der engen Grenzen des Urheberrechtsgesetzes ist ohne Zustimmung des Verlags unzulässig und strafbar. Das gilt insbesondere für Vervielfältigungen, Übersetzungen, Mikroverfilmungen und die Einspeicherung und Verarbeitung in elektronischen Systemen.

Höchste inhaltliche und technische Qualităt unserer Produkte ist unser Ziel. Bei der Produktion und Verbreitung unserer Bücher wollen wir die Umwelt schonen: Dieses Buch ist auf säurefreiem und chlorarm gebleichtem Papier gedruckt. Die Einschweißfolie besteht aus Polyăthylen und damit aus organischen Grundstoffen, die weder bei der Herstellung noch bei der Verbrennung Schadstoffe freisetzen.

Die Wiedergabe von Gebrauchsnamen, Handelsnamen, Warenbezeichnungen usw. in diesem Werk berechtigt auch ohne besondere Kennzeichnung nicht zu der Annahme, daß solche Namen im Sinne der Warenzeichen- und Markenschutz-Gesetzgebung als frei zu betrachten wären und daher von jedermann benutzt werden dürtten. 


\section{Vorwort}

Bei Unternehmen, die physisch greifbare und leicht differenzierbare Güter herstellen, ergeben sich assoziierte und zugeschätzte Eigenschaften oft aus dem Produkt, d. h. sie lassen sich meist direkt ableiten. Bei Dienstleistungen und insbesondere bei Dienstleistungen um das Geld, dem neutralsten aller Güter, sind die assoziierten und zugeschătzten Qualităten weniger stringent vorgegeben. Das einzelne Bankprodukt kann sich in der Regel nicht als eigenständige Marke entwickeln, vielmehr erhält die Institution als Ganzes ein Image, das der Unternehmung und deren Dienstleistung eine Markenqualität und ein Markenimage zuordnet. Image und Ruf, die sich in der Etablierung einer „Marke“ manifestieren, können für den Erfolg am Markt entscheidend sein.

In der vorliegenden Studie analysiert Markus Weber die Markenbildung und Markenpolitik nicht nur detailliert, sondern präsentiert sie auch informativ und interessant. Bei inm kann man nachvollziehen, daß die Kreation einer Marke sowohl eine spannende Marketingaufgabe als auch entscheidend für den Erfolg sein kann. Die besondere Verbindung zwischen der Leistung und dem Image bei der Markenpolitik einer Bank stellt der Autor auch im Zusammenhang mit der gewählten Strategie und dem dafür geeigneten Instrumentarium dar und bietet damit auch dem Praktiker viele wertvolle Anregungen.

New York/Frankfurt am Main

Februar 1992 


\section{VORWORT}

In der absatzwirtschaftlichen Literatur hat die Erörterung markenpolitischer Themen eine lange Tradition. Jedoch beschränkte sich die Diskussion über Jahrzehnte hinweg auf die Markenpolitik von Konsumgüterherstellern. Dienstleistungsunternehmen wurden meist wenig Chancen eingeräumt, Marken zur Erreichung absatzpolitischer Ziele zu instrumentalisieren. Gegenüber Kreditinstituten waren solche Vorbehalte besonders ausgeprägt, denn das Image des stillen und diskreten Bankiers unterschied sich diametral von der aufsehenerregenden Reklame, mit der Konsumgüterhersteller und Händler um die Gunst der Nachfrager kämpften.

Infolge verschärften Wettbewerbs haben Kreditinstitute in zunehmendem Umfang die Notwendigkeit erkannt, sich gegenüber konkurrierenden Unternehmen - seien es andere Banken oder sogenannte Non- und Near-banks - zu profilieren, ihre spezifische Kompetenz herauszustellen und die Zielpersonen von der Vorteilhaftigkeit ihrer Dienstleistungen zu überzeugen. Zur Erreichung dieses Ziels kann Markenpolitik einen wichtigen Beitrag leisten.

Die vorliegende Arbeit, die von der Fakultät für Betriebswirtschaftslehre der Universität Mannheim als Dissertation angenommen wurde, soll den Leserinnen und Lesern einen Überblick über markenpolitische Ziele, Strategien und Instrumente von Bankunternehmungen geben. Sie richtet sich sowohl an Studierende als auch an Praktiker in Kreditinstituten und Verbänden.

Herzlichen Dank schulde ich den vielen Menschen, die mich mit Informationen, Ratschlägen, Diskussionsbeiträgen und technischen Dienstleistungen unterstützt und auf diese Weise zur Fertigstellung der Arbeit beigetragen haben. Stellvertretend für alle danke ich Eva Wunsch, der ich diese Arbeit widme.

Mannheim, im Februar 1992

Markus Weber 


\title{
MARK ENPOLITIK DES BANKBETRIEBS
}

\author{
IN H A L TS VERZEICHN IS
}

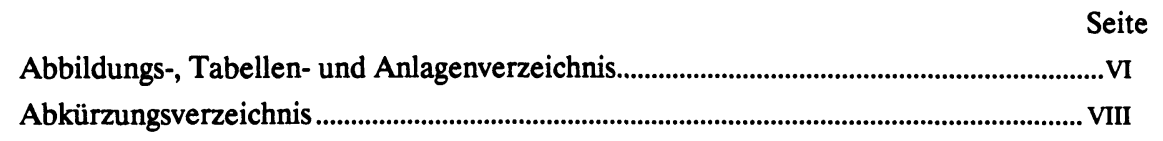

1. Einleitung............................................................................................................. 1

1.1 Markenpolitik als Profilierungskonzept im Wettbewerb ........................................... 1

1.2 Themenauffassung und -abgrenzung............................................................................. 3

1.2.1 Wissenschaftstheoretische Grundlagen......................................................... 3

1.2.2 Objektbereich der Untersuchung......................................................................... 4

1.3 Aufbau der Arbeit und Gang der Untersuchung.......................................................... 5

2. Terminologische Grundlagen und Erläuterungen .............................................................. 7

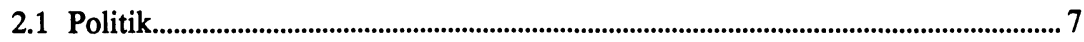

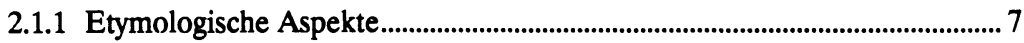

2.1.2 Klassifikatorische Aspekte .................................................................................. 10

2.1.3 Instrumentell-teleologische Aspekte ............................................................... 11

2.1.4 Interaktionistische Aspekte............................................................................... 13

2.1.4.1 Systemaspekt........................................................................................... 13

2.1.4.2 Konkurrenzaspekt................................................................................. 15

2.1.4.3 Machtaspekt........................................................................................ 17

2.2 Marke................................................................................................................ 20

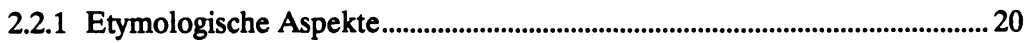

2.2.1.1 Semantische Ebene............................................................................. 20

2.2.1.2 Konzeptuelle Ebene .........................................................................21

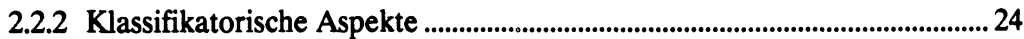

Exkurs: Marke und Markenartikel .................................................................2 27

2.2.3 Instrumentell-teleologische Aspekte ..............................................................2 28

Exkurs: Wertneutraler und teleologischer Qualitätsbegriff ..........................30

2.2.4 Juristische Aspekte ................................................................................................ 31

2.2.4.1 Warenzeichen und Dienstleistungsmarken ........................................33

2.2.4.2 Firmennamen und Unternehmensbezeichnungen ............................ 33

2.2.4.3 Aktuelle Probleme...............................................................................34

2.2.4.3.1 Markenpiraterie...................................................................... 34

2.2.4.3.2 Harmonisierung des Markenrechts in der Europäischen Gemeinschaft ..............................................................35

2.2.4.3.3 Bilanzierung von Markenzeichen ....................................... 36 


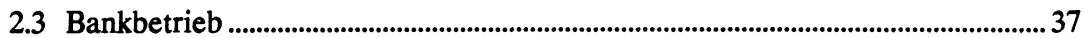

2.3.1 Funktional-transformatorische Klassifikation...................................................38

2.3.2 Organisatorisch-institutionelle Klassifikation ................................................. 42

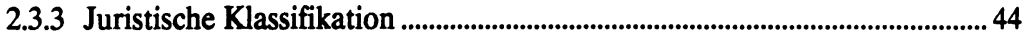

2.3.4 Systemorientierter Bankbegriff ........................................................................ 45

2.4 Definition der Markenpolitik des Bankbetriebs...........................................................46

3. Die Bedeutung der Bankloyalität für die bankbetriebliche Markenpolitik................... 47

3.1 Grundlegende Bemerkungen zu Treue und Loyalität.................................................. 47

3.2 Bankloyalität als Sonderform der Treue...................................................................... 49

3.3 Theoretische Ansätze zur Erklärung von Kundentreue und Bankloyalität ..........51

3.3.1 Der lerntheoretische Ansatz von Süchting ......................................................51

3.3.2 Der lerntheoretische Ansatz von Wiswede........................................................53

3.3.3 Dissonanz- und risikotheoretische Ansätze von Starkl und Kurz .................54

3.3.4 Das Operatorenmodell von Kuehn...................................................................5

3.3.5 Der entscheidungstheoretische Ansatz von Kroeber-Riel.............................57

3.3.6 Der produkttypologische Ansatz von Kumar.................................................58

3.4 Sozialpsychologische Aspekte der Bankloyalität............................................................59

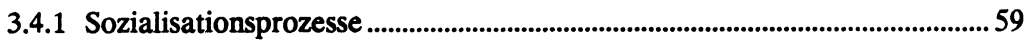

3.4.2 Orientierung an Referenzgruppen und Meinungsführern .............................6 60

3.4.3 Einstellungen ............................................................................................................ 62

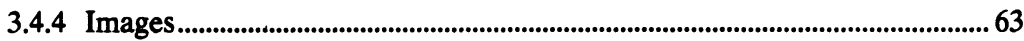

3.4.5 Wahrgenommenes Risiko........................................................................................... 63

4. Markenpolitik im Kontext strategischen Bankmanagements .............................................64

4.1 Strategisches Management als Führungskonzeption................................................64 64

4.1.1 Das Situationsmodell als heuristische Basis zur Strategieentwicklung...... 64

4.1.1.1 Die Vertriebssituation...........................................................................65

4.1.1.1.1 Spezifika der Bankdienstleistung........................................66

4.1.1.1.2 Spezifika des Kreditinstituts, insbesondere Personalaspekte........................................................................66

4.1.1.2 Die Marktsituation.......................................................................................... 69

4.1.1.2.1 Veränderungen im ökonomischen Umfeld..................... 69

4.1.1.2.2 Verschiebungen in der demographischen Struktur .......71

4.1.1.2.3 Veränderungen im gesellschaftlichen Wertespektrum ....................................................................... 73

4.1.1.2.4 Innovationen im technologischen Bereich ....................... 74

4.1.1.2.5 Anpassungen der rechtlichen Rahmenbedingungen .... 75

4.1.2 Das Phasenkonzept des strategischen Managements in Kreditinstituten......................................................................................................... 76

4.2 Theoretische Ansătze zur Erklärung des Markenphänomens ................................. 80

4.2.1 Markenbildung als Instrument zur Verringerung von Informations. asymmetrie

4.2.2 Markenphilosophie versus Philosophie der Marke......................................... 84 
4.3 Markenpolitische Ziele......................................................................................... 87

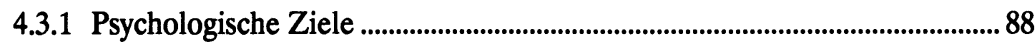

4.3.1.1 Individuation und Identifikation......................................................... 88

4.3.1.2 Aufbau und Pflege von Marken- und Institutstreue........................ 90

4.3.1.3 Aufbau und Festigung des innerbetrieblichen Zusammengehörigkeitsgefühls ...................................................................................... 91

4.3.2 Ökonomische Ziele ........................................................................................92

4.3.2.1 Marktbezogene Ziele .......................................................................... 93

4.3.2.2 Betriebsbezogene Ziele ..................................................................... 94

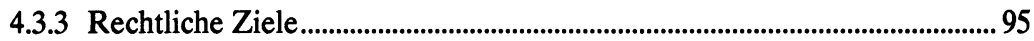

4.3.3.1 Schutz gegen Nachahmung..............................................................96

4.3.3.2 Erwerb und Vergabe von Nutzungsrechten.....................................98

4.4 Markenpolitische Strategien ................................................................................... 100

4.4.1 Institutionell orientierte Strategien ........................................................... 101

4.4.1.1 Markenstrategien auf Einzelproduktebene ..................................... 102

4.4.1.2 Markenstrategien auf Produktgruppenebene.................................. 103

4.4.1.3 Markenstrategien auf Unternehmensebene .................................... 105

4.4.1.4 Markenstrategien auf Institutsgruppenebene................................... 106

4.4.2 Funktionell orientierte Strategien............................................................... 109

4.4.2.1 Zweitmarken-Strategie .................................................................... 109

4.4.2.2 Markentransfer-Strategie.................................................................. 111

4.4.3 Die Strategietypen Porters als Klassifikationsraster für markenpolitische Strategien ............................................................................................. 113

4.4.4 Idealtypische Strategien bankbetrieblicher Markenpolitik.......................... 115

4.4.4.1 Die Strategie der Discount-Bank ...................................................... 116

4.4.4.2 Die Strategie der Experten-Bank ...................................................... 117

4.4.4.3 Die Strategie der Komfort-Bank ......................................................118

4.4.4.4 Die Strategie der Exklusiv-Bank ....................................................... 118

4.4.4.5 Die Strategie der Philanthropen-Bank ............................................. 120

4.4.4.6 Die Strategie der Technik-Bank ........................................................ 120

4.5 Markenpolitische Instrumente ......................................................................... 121

4.5.1 Originäre markenpolitische Instrumente..................................................... 122

4.5.1.1 Markenname............................................................................... 122

4.5.1.1.1 Semiotische Aspekte........................................................... 123

4.5.1.1.2 Messung der Aufmerksamkeitswirkung.......................... 125

4.5.1.1.3 Typenbildende Kriterien ................................................... 127

4.5.1.2 Markenzeichen ................................................................................... 130

4.5.1.2.1 Form ............................................................................... 131

4.5.1.2.2 Farbe ................................................................................ 133

4.5.2 Derivative markenpolitische Instrumente ….............................................. 135

4.5.2.1 Instrumente der Imagepolitik ....................................................... 136

4.5.2.1.1 Äußeres Erscheinungsbild ............................................... 137

4.5.2.1.2 Mitarbeiterverhalten.......................................................... 139 


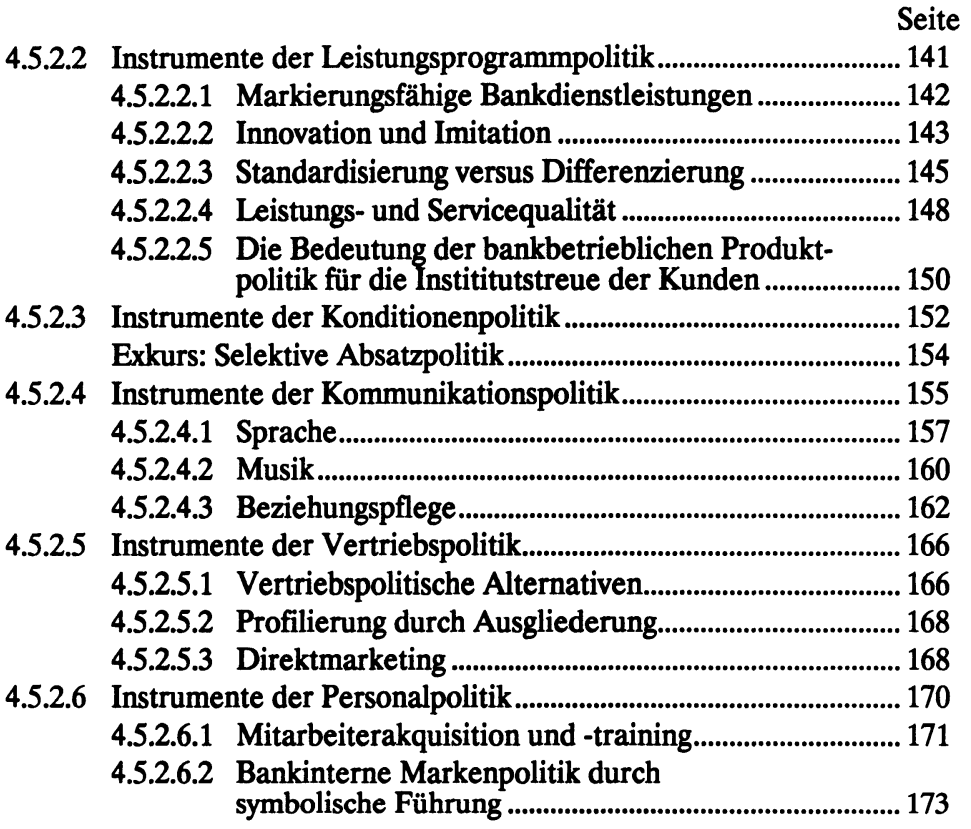

5. Umfrage zur Markenpolitik in Geldinstituten................................................................. 175

5.1 Notwendigkeit und Zielsetzung der Umfrage........................................................ 175

5.2 Untersuchungsdesign ................................................................................................. 176

5.2.1 Bestimmung der Stichprobe ...................................................................... 176

5.2.2 Gestaltung des Fragebogens ...................................................................... 177

5.2.2.1 Formale Gestaltung........................................................................... 177

5.2.2.2 Inhaltliche Gestaltung........................................................................ 178

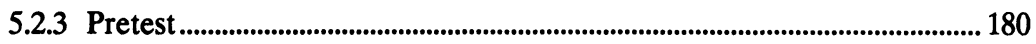

5.3 Auswertung der zurückerhaltenen Fragebögen.................................................... 181

5.3.1 Strukturelle Zusammensetzung der Stichprobe .............................................. 181

5.3.2 Ermittlung der Ergebnisse zu den einzelnen Fragen................................... 181

5.4 Ergebnisse der Umfrage ...................................................................................... 182

5.4.1 Einflüsse auf die Bereitschaft der Kunden zur Inanspruchnahme von Bankdienstleistungen ....................................................................................... 182

5.4.2 Kriterien für die Inanspruchnahme von Bankdienstleistungen.................. 183

5.4.3 Die Bedeutung von 15 markenpolitischen Erfolgsfaktoren für die Bereitschaft zur Leistungsinanspruchnahme................................................... 184

5.4.3.1 Produkt- und servicebedingte Erfolgsfaktoren ............................... 187

5.4.3.1.1 Originalität der Dienstleistungen .................................... 187

5.4.3.1.2 Servicequalität ...................................................................... 188

5.4.3.1.3 Breite der Leistungspalette und Grad der Spezialisierung ...................................................................... 188

5.4.3.1.4 Zins- und Provisionssätze.................................................... 189 
5.4.3.2 Institutsbedingte Erfolgsfaktoren

Seite

5.4.3.2.1 Institutsimage und -erscheinungsbild 189

5.4.3.2.2 Fachwissen 189

5.4.3.2.3 Freundlichkeit und Motivation der Mitarbeiter........... 190

5.4.3.2.4 Originalität der Kommunikationspolitik ........................ 191

5.4.3.2.5 Standort.............................................................................. 192

5.4.3.2.6 Organisationsstruktur und technische Ausstattung .... 192

5.4.3.3 Kosten- und Konkurrenzintensität. 193

5.4.3.4 Beeinträchtigung der Kundenzufriedenheit durch Mängel der Bank

5.4.4 Soziodemographische Aspekte ....................................................................... 196

5.4.4.1 Alter ..................................................................................................... 197

5.4.4.2 Bildungsgrad ................................................................................................ 199

5.4.4.3 Einkommen ....................................................................................... 199

5.4.4.4 Wohngegend ........................................................................................... 200

5.4.5 Verbreitung markenpolitischer Konzepte in der bankbetrieblichen

Praxis...........................................................................................................202

5.4.5.1 Reichweite der Markenpolitik ..........................................................202

5.4.5.2 Stellenwert der Markenpolitik..........................................................202

5.4.5.3 Nutzung markenpolitischer Instrumente..........................................203

5.5 Differenzierung der Umfrageergebnisse durch die Bildung verschiedener

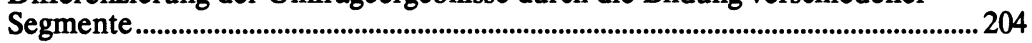

5.6 Entwurf eines Modells zur Bewertung markenpolitischer Erfolgsfaktoren....... 209

5.6.1 Markenpolitische Erfolgsfaktoren im Geschäft mit Mengenkunden........211

5.6.2 Markenpolitische Erfolgsfaktoren im Geschäft mit vermögenden

Privatkunden....................................................................................................213

5.6.3 Markenpolitische Erfolgsfaktoren im Geschäft mit Firmenkunden .........214

5.6.4 Kritik des Modells...............................................................................................216

6. Ableitung markenpolitischer Tendenzen und Empfehlungen ......................................218

6.1 Relativierung des normativen Anspruchs................................................................ 218

6.2 Prüfung der Sinnhaftigkeit einer Markenpolitik .................................................219

6.3 Formulierung des ideellen Kerns einer Marke....................................................... 220

6.4 Auswahl von Markennamen und Markenzeichen.................................................. 224

6.5 Zum "make or buy" markentechnischer Dienstleistungen ......................................225

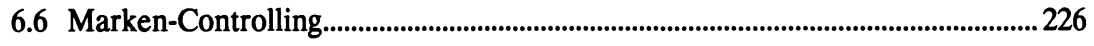

7. Zusammenfassung und Ausblick.................................................................................. 228

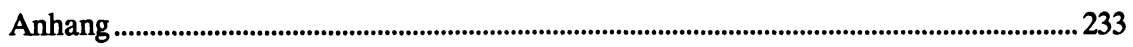

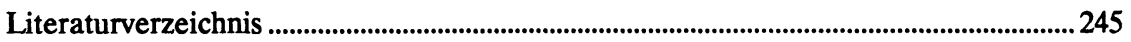

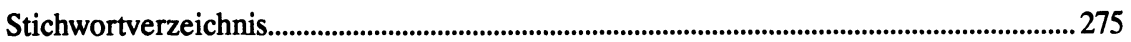




\section{ABBILDUNGS-, TABELLEN- UND ANLAGENVERZEICHNIS}

Seite

Abbildung 1: Lernkurve der Bankloyalität ....................................................................5 52

Abbildung 2: Zusammenhang zwischen Wiedernutzungsabsicht und kognitiver

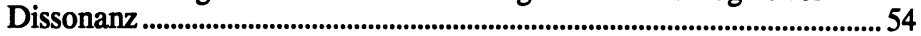

Abbildung 3: Kumulatives Lernmodell .........................................................................56

Abbildung 4: Das Situationsmodell des Bankbetriebs..................................................... 77

Abbildung 5: Sphären gewerblichen Rechtsschutzes ....................................................... 96

Abbildung 6: Wissenserwerb und Wissensanwendung in verschiedenen Vertriebskonzepten .........................................................................................99

Abbildung 7: Einordnung markenpolitischer Strategien in das Strategietypenschema nach Porter ........................................................................................ 115

Abbildung 8: Ebenen der Semiotik.................................................................................. 124

Abbildung 9: Änderung des Markenzeichens der Landeskreditbank BadenWürttemberg.

Abbildung 10: Einflüsse auf die Bereitschaft zur Inanspruchnahme von Bankdienstleistungen .................................................................................. 183

Abbildung 11: Kriterien für die Inanspruchnahme von Bankdienstleistungen.............. 184

Abbildung 12: Bedeutung von 15 Erfolgsfaktoren für Mengen-, vermögende Privatkunden und Firmenkunden .

Abbildung 13: Kostenintensität von 15 markenpolitischen Erfolgsfaktoren.................. 193

Abbildung 14: Konkurrenzintensität von 15 markenpolitischen Erfolgsfaktoren........ 194

Abbildung 15: Verärgerungsempfindlichkeit verschiedener Kundengruppen bei Mängeln der Bank

Abbildung 16: Soziodemographische Einflüsse auf die Markenorientierung von Bankkunden ...................................................................................................... 196

Abbildung 17: Reichweite der Markenpolitik.................................................................... 202

Abbildung 18: Stellenwert der Markenpolitik.................................................................. 203

Abbildung 19: Markenpolitische Instrumente................................................................ 204

Abbildung 20: Gesamtbewertung der Erfolgsfaktoren für das Segment "Mengenkunden".

Abbildung 21: Gesamtbewertung der Erfolgsfaktoren für das Segment "vermögende Privatkunden"

Abbildung 22: Gesamtbewertung der Erfolgsfaktoren für das Segment "Firmenkunden".

Abbildung 23: Beispiele für Markenzeichen von Kreditinstituten ..................................234 
Tabelle 1: Bedeutung von 15 Erfolgsfaktoren für verschiedene Kundengruppen

Tabelle 2: Richtung soziodemographischer Einflüsse auf die Markenorientierung von Bankkunden.

Tabelle 3: Markenpolitische Erfolgsfaktoren im Geschäft mit Mengenkunden

Tabelle 4: Markenpolitische Erfolgsfaktoren im Geschäft mit vermögenden Privatkunden.

Tabelle 5: $\quad$ Markenpolitische Erfolgsfaktoren im Geschäft mit Firmenkunden

Anlage 1: $\quad$ BASIC-Programm zur Umwandlung von Buchstaben in ihre relativen Informationswerte

Anlage 2: $\quad$ Titelblatt des Fragebogens .............................................................................236

Anlage 3: $\quad$ BASIC-Programm zur Berechnung von Indexwerten................................ 237

Anlage 4: $\quad$ Ergebnisse der Umfrage zur Markenpolitik................................................ 238

Anlage 5: $\quad$ BASIC-Programm zur Berechnung von Multiplikatoren .......................... 242

Anlage 6: $\quad$ Nicht alltägliche Beispiele für markenpolitische Strategien und ihre Resultate - einige "Lesefrüchte" 


\section{ABKÜRZUNGSVERZEICHNIS}

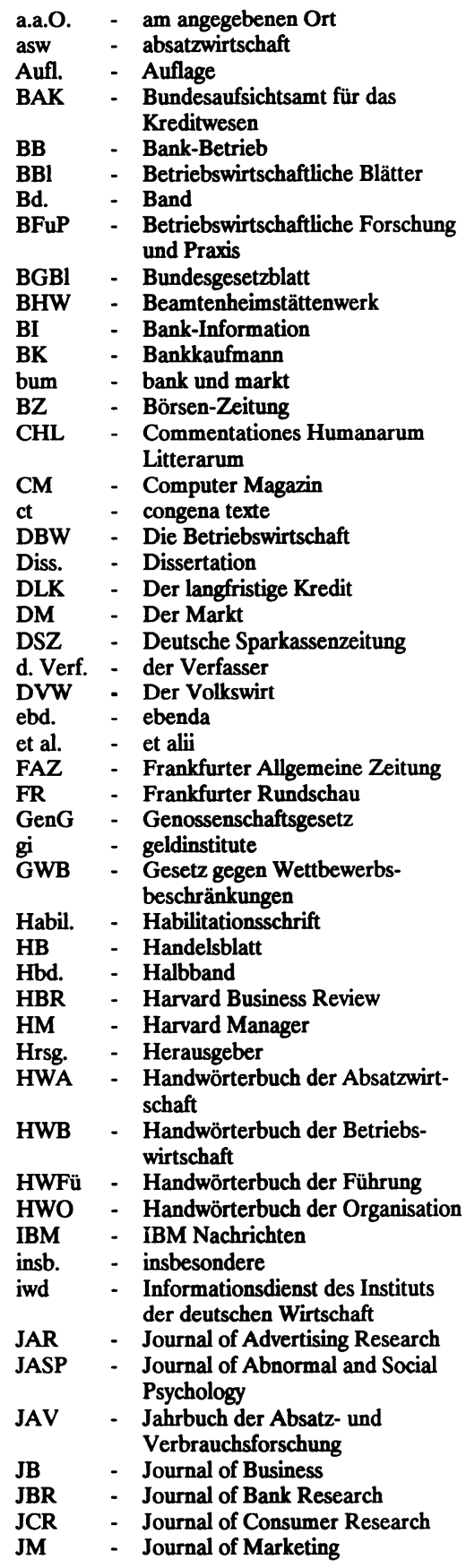

JMR - Journal of Marketing Research

KKB - Kundenkreditbank

KMB - Köln-Mannheimer Beiträge zur Wirtschafts- und Organisationspsychologie

KuK - Kredit und Kapital

KWG - Gesetz über das Kreditwesen

KWS - Kleiner Wirtschaftsspiegel

LBS - Landesbausparkasse

MA - Markenartikel

m.a.W. - mit anderen Worten

MB - Mannheimer Berichte

MJ - Marketing Journal

MM - Mannheimer Morgen

mm - manager magazin

Mrd. - Milliarden

MZFP - Marketing - Zeitschrift für Forschung und Praxis

NCSS - Number Cruncher Statistical System

Nr. - Nummer

o.J. - ohne Jahresangabe

o.O. - ohne Ortsangabe

o.V. - ohne Verfasserangabe

ÖBA - Österreichisches Bank-Archiv

PH - Psychologie heute

PrPG - Gesetz zur Bekämpfung der Produktpiraterie

RB - Rheinisches Genossenschaftsblatt

RZ - Rhein-Zeitung

S. - Seite

SI - Sparkassen International

Sp. - - Spalte

SWD - Sparkassen-Werbedienst

TBM - The Bankers Magazine

u.a. - und andere

UWG - Gesetz gegen den unlauteren

vgl. - vergleiche

Vol. - Volume

WD - Wirtschaftsdienst

WiSt - Wirtschaftswissenschaftliches Studium

WISU - Das Wirtschaftsstudium

WW - Wirtschaftswoche

WZG - Warenzeichengesetz

ZfB - Zeitschrift für Betriebswirtschaft

ZfbF - Zeitschrift für betriebswirtschaftliche Forschung

ZfgG - Zeitschrift für das gesamte Genossenschaftswesen

ZfgK - Zeitschrift für das gesamte Kreditwesen

ZfhF - Zeitschrift für handelswissenschaftliche Forschung

zfo - Zeitschrift Führung und Organisation

zit. - zitiert 